\title{
Upregulation of CPT1A is essential for the tumor- promoting effect of adipocytes in colon cancer
}

Xiaopeng Xiong ${ }^{1}$, Yang-An Wen ${ }^{1}$, Rachelle Fairchild ${ }^{1}$, Yekaterina Y. Zaytseva $\mathbb{E}^{2}$, Heidi L. Weiss ${ }^{1}$, B. Mark Evers $\mathbb{D}^{1,3}$ and Tianyan Gao (1,4

\begin{abstract}
Colon tumors grow in an adipose tissue-enriched microenvironment. Locally advanced colon cancers often invade into surrounding adipose tissue with a direct contact with adipocytes. We have previously shown that adipocytes promote tumor growth by modulating cellular metabolism. Here we demonstrate that carnitine palmitoyltransferase I (CPT1A), a key enzyme controlling fatty acid oxidation (FAO), was upregulated in colon cancer cells upon exposure to adipocytes or fatty acids. In addition, CPT1A expression was increased in invasive tumor cells within the adipose tissue compared to tumors without direct contact with adipocytes. Silencing CPT1A abolished the protective effect provided by fatty acids against nutrient deprivation and reduced tumor organoid formation in $3 \mathrm{D}$ culture and the expression of genes associated with cancer stem cells downstream of Wnt/ $\beta$-catenin. Mechanistically, CPT1A-dependent FAO promoted the acetylation and nuclear translocation of $\beta$-catenin. Furthermore, knockdown of CPT1A blocked the tumor-promoting effect of adipocytes in vivo and inhibited xenograft tumor initiation. Taken together, our findings identify CPT1A-depedent FAO as an essential metabolic pathway that enables the interaction between adipocytes and colon cancer cells.
\end{abstract}

\section{Introduction}

Altered metabolism has been recognized as a common hallmark of cancer ${ }^{1,2}$. Emerging evidence indicates that the tumor microenvironment (TME) is essential in shaping the landscape of cancer metabolism ${ }^{3}$. A profile of the components in the TME revealed that adipocytes constitute a major cell type that is abundantly associated with tumor cells ${ }^{4}$. Metastatic colon cancer cells often encounter adipocytes as they first disseminate from their primary tumor site. Previous studies indicated that cancer-associated adipocytes promote tumor growth and progression by secreting growth factors and proinflammatory cytokines into the $\mathrm{TME}^{5,6}$. However, it is less

Correspondence: Tianyan Gao (tianyan.gao@uky.edu)

${ }^{1}$ Markey Cancer Center, University of Kentucky, Lexington, KY 40536-0679, USA ${ }^{2}$ Department of Toxicology and Cancer Biology, University of Kentucky,

Lexington, KY 40536-0679, USA

Full list of author information is available at the end of the article

Edited by A. Stephanou understood how the direct interaction with adipocytes alters tumorigenic properties of cancer cells.

Recently, it has been shown that lipids produced in adipocytes can be transferred to cancer cells to promote tumor growth in ovarian cancer models, suggesting that local adipose tissues may have a direct role in supporting cancer cells ${ }^{7}$. Additionally, we reported that the uptake of fatty acids from adipocytes allows colon cancer cells to survive nutrient deprivation conditions by upregulating mitochondrial fatty acid oxidation (FAO $)^{8}$. This transfer of fatty acids from adipocytes to cancer cells has been demonstrated in breast and melanoma cancer models as well $^{9,10}$. Interestingly, the presence of cancer cells stimulates the release of fatty acids by promoting lipolysis in adipocytes, thus indicating a two-way communication between cancer cells and adipocytes in the $\mathrm{TME}^{7,8}$. Furthermore, treatment with fatty acids enhances the expression of genes associated with colon cancer stem cells (CSCs) and suppresses genes associated with intestinal epithelial cell differentiation ${ }^{8}$. This finding is

\section{(c) The Author(s) 2020}

(c) Open Access This article is licensed under a Creative Commons Attribution 4.0 International License, which permits use, sharing, adaptation, distribution and reproduction cc) in any medium or format, as long as you give appropriate credit to the original author(s) and the source, provide a link to the Creative Commons license, and indicate if changes were made. The images or other third party material in this article are included in the article's Creative Commons license, unless indicated otherwise in a credit line to the material. If material is not included in the article's Creative Commons license and your intended use is not permitted by statutory regulation or exceeds the permitted use, you will need to obtain permission directly from the copyright holder. To view a copy of this license, visit http://creativecommons.org/licenses/by/4.0/. 
consistent with the notion of CSC plasticity in that nonCSCs are capable of converting to CSCs given the right cue presented by the $\mathrm{TME}^{11-13}$. Taken together, these studies suggest that the close interaction between adipocytes and cancer cells plays an important role in regulating cancer metabolism.

Although the transfer of fatty acids from adipocytes to cancer cells has been confirmed in several studies ${ }^{7-10}$, the molecular mechanism underlying fatty acidsdependent metabolic regulation in cancer cells remains elusive. Here we further determined the role of CPT1A, a rate-limiting enzyme required for mitochondrial FAO, in mediating the tumor-promoting effect of adipocytes in colon cancer. Using primary colon cancer cells, 3D tumor organoids and in vivo xenograft models, we showed that uptake of fatty acids promotes the expression of CPT1A through the activation of PPAR $\delta$. Consequently, knockdown of CPT1A attenuated fatty acid utilization and eliminated the pro-survival advantage provided by adipocytes. In addition, we identified $\beta$-catenin acetylation as a novel mechanism connecting upregulation of FAO with increased Wnt/ $\mathrm{W}$-catenin signaling. Together, results from our study provided new mechanistic insights into the tumor-promoting effect of adipocytes in colon cancer.

\section{Materials and methods Cells and reagents}

Patient-derived colon cancer PT130 cells were established as described previously ${ }^{8,14}$. Human colon cancer SW480 cells were purchased from ATCC. Both cell lines were maintained in DMEM supplemented with 10\% fetal bovine serum (FBS, Sigma-Aldrich) and $1 \%$ penicillin-streptomycin. The cell lines were authenticated using short tandem repeat (STR) DNA profiling and tested negative for mycoplasma contamination (Genetica). The shRNA-targeting sequences for human CPT1A are as the following: 5'- GCCATGAAGCTCTTAGACAAA-3' (C6), and $5^{\prime}$-CGATGTTACGACAGGTGGTTT- $3^{\prime}$ (C7); and for mouse Cpt1a is: 5'-GCTATGGTGTTTCCTACATTA- $3^{\prime}$. The following reagents were obtained from commercial sources as specified below: Acetyl-Coenzyme A Assay Kit, oleic acid (OA) (albumin complex), palmitic acid (PA), linoleic acid (LA) (albumin complex), octanoate, GW501516, GSK3787, and etomoxir (ETO) were from Sigma-Aldrich; BIODPY 493/503, N-2, and B-27 supplement were from Thermo Fisher Scientific. Bovine serum albumin (BSA)-conjugated palmitate was prepared according to the Seahorse protocol (Seahorse Bioscience). The concentrations of long chain fatty acids used in the cell treatment experiments $(100-200 \mu \mathrm{M})$ are below the average plasma fatty acid concentrations found in healthy people $^{15}$. For fatty acid treatment experiments, cells were cultured in low glucose media $(5 \mathrm{mM}$, which is close to the normal blood glucose level) supplemented with $10 \%$ lipoprotein-deficient bovine serum (Alfa Aesar, J65182AMG). The octanoate concentration ( $3 \mathrm{mM})$ used for treating cells was based on the amount of octanoate needed to induce the expression genes related to "fatty acid metabolic process" as described previously ${ }^{16}$.

\section{Isolation of human mature adipocytes}

Human omental or mesenteric fat tissues were collected from colon cancer patients undergoing surgery at the University of Kentucky Markey Cancer Center. The process for patients' material collection was approved by the University of Kentucky's Office for the Protection of Human Subjects. The isolation of adipocytes was carried out as described previously ${ }^{8}$. Equal amount of adipocytes were used as determined by the packed cell volume in all experiments (the density of purified adipocytes used is $\sim 1-2 \times 10^{6}$ cells $\left./ \mathrm{ml}\right)$.

\section{Immunohistochemical (IHC) staining}

Paraffin-embedded colon cancer patient specimens were obtained from the Biospecimen Procurement and Translational Pathology Shared Resource Facility of the Markey Cancer Center. The diagnosis and staging of each cancer case were confirmed by pathologist. IHC staining of paraffin-embedded tissue sections was performed as previously described $^{8,17}$. The CPT1A antibody (\#12252) was obtained from Cell Signaling. The stained sections were visualized using a Nikon Eclipse 80i upright microscope. To quantify the relative CPT1A expression levels, pixel intensity values were used to define the percentage of tumor cells with positive staining using the HALO image analysis platform (Indica Labs).

\section{Measurements of cellular acetyl-CoA (Ac-CoA) levels}

The cellular levels of Ac-CoA were determined using the Acetyl-Coenzyme A Assay Kit (Sigma-Aldrich). Briefly, cells were cultured in low glucose media supplemented with $10 \%$ FBS. Total of $10^{6}$ cells were trypsinized, lysed in perchloric acid and neutralized using $\mathrm{KHCO}_{3}$. After centrifuging at $13,000 \times g$ for $10 \mathrm{~min}$, supernatants were collected and used for Ac-CoA measurements. The protein contents in the pellets were determined using the BCA Protein Assay Kit. The levels of Ac-CoA were presented as $\mathrm{pmol} / \mathrm{mg}$ of protein.

\section{Seahorse extracellular flux analysis}

The Seahorse XF96 Extracellular Flux Analyzer (Agilent) was used to measure the respiration activity of colon cancer cells as described previously ${ }^{8,14,18}$. The mitochondrial stress tests were performed according to manufacturer's protocol. The relative levels of basal and maximal respiration were calculated based on OCR data obtained in the Mito stress tests. The FAO assays were 
performed as previously described with the following modifications ${ }^{8}$. Briefly, cells were seeded at the density of $3 \times 10^{4}$ cells per well in a XF96 plate and subsequently incubated in substrate-limited medium (DMEM with $0.5 \mathrm{mM}$ glucose, $1.0 \mathrm{mM}$ glutamine, $0.5 \mathrm{mM}$ carnitine, and $1 \%$ FBS) for $\sim 16 \mathrm{~h}$. Prior to the beginning of FAO measurements, the medium was switched into FAO assay medium $(200 \mu \mathrm{M}$ palmitate-BSA, $111 \mathrm{mM} \mathrm{NaCl}, 4.7 \mathrm{mM}$ $\mathrm{KCl}, 2.0 \mathrm{mM} \mathrm{MgSO}{ }_{4}, 1.2 \mathrm{mM} \mathrm{Na}_{2} \mathrm{HPO}_{4}, 2.5 \mathrm{mM}$ glucose, $0.5 \mathrm{mM}$ carnitine, and $5 \mathrm{mM}$ HEPES$)$. FCCP $(3 \mu \mathrm{M})$, ETO $(200 \mu \mathrm{M})$, and antimycin $\mathrm{A}(4 \mu \mathrm{M})$ were added subsequently at the indicated time. For measurements using OA-BSA as substrate, OA-BSA $(100 \mu \mathrm{M})$ was added to the FAO assay medium replacing palmitate-BSA. All OCR measurements were normalized to total protein contents in each well. The basal, maximum, and reserved FAO were calculated based on OCR measurements upon the addition of mitochondrial inhibitors.

\section{Fatty acid degradation assay}

Cells were seeded in 96-well plates at a density of $1 \times$ $10^{4}$ cells per well and incubated with OA $(200 \mu \mathrm{M})$ overnight. The cells were then switched to low glucose medium supplemented with 10\% FBS and fixed in paraformaldehyde at indicated time points. To quantify the amount of lipids, the fixed cells were stained with BODIPY $493 / 503(1 \mu \mathrm{g} / \mathrm{ml})$ and the amount of BODIPY fluorescence was measured in cell lysates using a SpectraMax M5 Microplate Reader. For visualizing cellular lipid contents, cells were seeded onto coverslips in six-well plates, loaded with OA and allowed to unload for 24-48 h. The cells were then fixed and stained with BODIPY 493/503 and DAPI. The immunofluorescence images were obtained using a Nikon $\mathrm{A}^{+}$confocal microscope.

\section{Tumor organoid formation assay}

Tumor organoids generated from Apc/Kras double mutant mice were cultured as described previously ${ }^{8}$. To silence Cpt1a expression, tumor organoids were dissociated into small cell clusters using TrypLE (Thermo) and incubated with sh-Cpt1a lentivirus in suspension for $6 \mathrm{~h}$ in a $37^{\circ} \mathrm{C}$ incubator. Cells were subsequently embedded in 50\% Matrigel in 3D growth medium (Advanced DMEM/F12 supplemented with $1 \times$ Glutamax, $1 \times \mathrm{N}-2,1 \times \mathrm{B}-27,1 \mathrm{mM} \mathrm{N}$-acetyl-L-cysteine, and $1 \%$ penicillin/streptomycin), and puromycin was added 2 days later to select for stable knockdown cells. To determine the tumor initiation capacity, organoids were dissociated to single cell suspensions using Accumax (Sigma-Aldrich). Total of 1000 cells per group were embedded in Matrigel as described above. The number of tumor organoids formed after 6 days were counted and analyzed. For gene expression analysis, tumor organoids were cultured in 3D Matrigel for 3 days and collected for RT-PCR ${ }^{14}$.

\section{Western blot analysis}

Colon cancer cells or tumor organoids were collected and detergent-solubilized cell lysates were obtained as described previously ${ }^{8,14}$. The NE-PER Nuclear and Cytoplasmic Extraction Kit (Thermo) was used to separate cytoplasmic and nuclear fractions. Equal amounts of total cell lysates were resolved by SDS-PAGE and subjected to Western blot analysis. The following antibodies, including CPT1A, acetylated-lysine (pan-Lys, \#9441) acetyl-histone H3 (Lys9) (\#9649), acetyl-histone H3 (Lys27) (\#8173), histone H3 (\#14269), active- $\beta$-catenin (\#8814), total $\beta$-catenin $(\# 8480)$, were purchased from Cell Signaling; the acetyl- $\alpha$-tubulin (T7451) and $\beta$-actin (A1978) antibodies were from Sigma-Aldrich; and the total $\alpha$-tubulin (sc-5286) and Lamin A/C (sc-20681) antibodies were from Santa Cruz.

\section{Quantitative RT-PCR}

Total mRNAs were isolated from cells using the RNeasy Mini Kit (Qiagen). Equal amounts of RNA were subjected to the reverse transcription PCR with the High Capacity cDNA Reverse Transcription kit (Thermo). The cDNAs obtained were subjected to RT-PCR reactions using the SYBR Green Master Mix (Thermo) and primers listed in Supplementary Table S1. To determine the expression of human and mouse CPT1A, gene-specific probes were purchased from Thermo and used in RT-PCR reactions using Taqman Gene Expression Master Mix (Thermo). All values were normalized to the level of $\beta$-actin.

\section{Xenograft tumorigenesis}

All animal procedures were done using protocols approved by the University of Kentucky Animal Care and Use Committee. Six to eight week-old NOD.Cg-Prkdc ${ }^{\text {scid }}$ $\mathrm{IL} 2 \mathrm{rg}^{\mathrm{tm} 1 \mathrm{Wjl}} / \mathrm{SzJ}$ (NSG, The Jackson Laboratory) mice were used. Both male and female mice of equal numbers were included in each group. Mice were housed in barrier rooms with 12-h light/dark cycle. For tumor growth assay, control and CPT1A knockdown SW480 cells in 5\% Matrigel suspension $\left(5 \times 10^{5}\right.$ cells in $\left.100 \mu \mathrm{l}\right)$ were mixed with $50 \mu \mathrm{l}$ of freshly isolated human adipocytes (contain $\sim 100,000$ adipocytes) or PBS and inoculated subcutaneously. The tumor size was measured every week with a caliper, and the tumor volume was defined as (longest diameter) $\times(\text { shortest diameter })^{2} / 2$. At the end of experiments, tumors were harvested and subjected to mRNA and protein analysis. For tumor initiation assay, 100 or 1000 SW480 cells were mixed with Matrigel and adipocytes as described above and injected subcutaneously. The number of tumors formed was determined 3 months post injection. 


\section{Statistical analysis}

In experiments to assess rate of FAO, relative cell survival, mRNA expression, levels of Ac-CoA and colony formation were summarized using bar graphs and pairwise comparisons between different conditions were carried out using two-sample $t$-tests. For measuring the time course of fatty acid degradation, one-way or two-way analysis of variance models with two-way interaction terms for experimental factors such as treatment group, cell types and time were utilized. A linear mixed model was employed to compare slope of tumor volume growth curves over time between groups. The relative mRNA expression results represent average of three separate RT-PCR experiments with four replicates for each gene in each experiment. All other experiments were repeated three times and results shown represent the average of three experiments. Measurements of xenograft tumor growth were summarized at each time point of follow-up and analysis was performed using longitudinal models to account for repeatedly measured tumor volume over time within each mouse. The stem cell frequencies were calculated by extreme limiting dilution analysis (ELDA) ${ }^{19}$ using analytic tools available at http://bioinf. wehi.edu.au/ software/elda/.

\section{Results}

\section{CTP1A expression is upregulated by fatty acids in colon} cancer

We have shown previously that fatty acids released by adipocytes can be taken up by cancer cells to support cell survival by upregulating $\mathrm{FAO}^{8}$. Here we further investigated the mechanisms by which fatty acids induce this metabolic switch. To this end, we determined the expression of CPT1A in tumor specimens obtained from stage IV colon cancer patients. Interestingly, the expression of CPT1A was significantly increased in tumor cells that have invaded into the omental adipose tissue (adipocyte adjacent) compared to tumor cells without direct contact with adipocytes (primary tumors) (Fig. 1a-d and Supplementary Fig. S1). To examine the effect of fatty acids on CPT1A expression directly, we co-cultured PT130, a patientderived colon cancer cell line, and SW480 cells with adipocytes. The CPT1A expression was markedly increased in the presence of adipocytes as measured by quantitative RTPCR in colon cancer cells (Fig. 1e) and Western blotting analysis (Fig. 1f). Additionally, PT130 and SW480 cells were treated with OA, PA, or LA, three major fatty acid species found in human adipose tissues ${ }^{20}$, and the expression of CPT1A was consistently increased at both protein and mRNA levels (Fig. 1f, g). In subsequent experiments, OA or PA was used to examine CPT1Adependent effects in colon cancer cells. Together, these results suggest that upregulation of CPT1A may provide a necessary mechanism for cancer cells to adapt a fatty acidenriched microenvironment.
Silencing CPT1A alters cellular metabolism in colon cancer cells

Given that CPT1A is the first rate-limiting enzyme in FAO, we next investigated the effect of silencing CPT1A on cellular metabolism. Stable CPT1A knockdown PT130 and SW480 cells were generated using two different lentiviral shRNAs (Fig. 2a). To determine the rate of FAO, the oxygen consumption rates (OCRs) were measured by using palmitate as the metabolic substrate in control and CPT1A knockdown cells with Seahorse XF96 Extracellular Flux Analyzer (Fig. 2b-e). We found that the OCRs associated with basal, maximal. and reserved FAO were significantly decreased in CPT1A knockdown PT130 and SW480 cells (Fig. 2c, e), confirming the role of CPT1A in controlling mitochondrial FAO. Similar results were obtained using $\mathrm{OA}$ as the metabolic substrate in Seahorse measurements to confirm that knockdown of CPT1A decreases FAO in colon cancer cells (Supplementary Fig. S2). In addition, since FAO and glycolysis are two functionally coupled metabolic processes that can be utilized by cancer cells, we determined if knockdown of CPT1A alters mitochondria-dependent glucose metabolism using Seahorse analysis (Supplementary Fig. S3). Interestingly, when using glucose as the metabolic substrate, results from Seahorse Mito Stress Tests showed that the OCRs associated with both basal and maximal mitochondrial respiration as well as ATP production were significantly increased in CTP1A knockdown cells (Supplementary Fig. S3A-D). Moreover, measurements from Seahorse Glycolysis Tests indicated that the extracellular acidification rate (ECAR) was increased in CTP1A knockdown cells (Supplementary Fig. S3E-H). These results are consistent with previous reports that ETO treatment can increase glucose uptake in normal and malignant cells ${ }^{21,22}$. Taken together, our results suggest that downregulation of CPT1A impairs FAO in colon cancer cells. However, this decreased FAO can be compensated by increased glucose metabolism. Indeed, we did not observe significant changes in the rate of cell proliferation in CTP1A knockdown cells when cultured in regular growth medium containing sufficient supplies of glucose (Supplementary Fig. S4).

\section{Knockdown CPT1A impairs fatty acid utilization and cell survival under nutrient deprivation conditions}

To address the question if upregulation of CPT1A is necessary for cancer cells to utilize the exogenous fatty acids, control and CPT1A knockdown PT130 and SW480 cells were treated with OA for $24 \mathrm{~h}$ (the loading phase) and subsequently cultured in low glucose media for additional $24-48 \mathrm{~h}$ (the unloading phase) to allow the preferential utilization of fatty acids. Upon loading cells with OA, similar levels of lipid droplets accumulation were detected in control and CPT1A knockdown cells using 


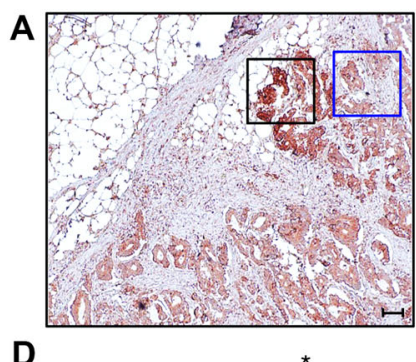

D

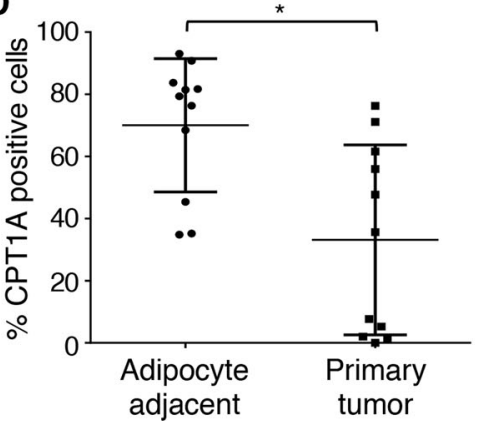

$\mathbf{F}$

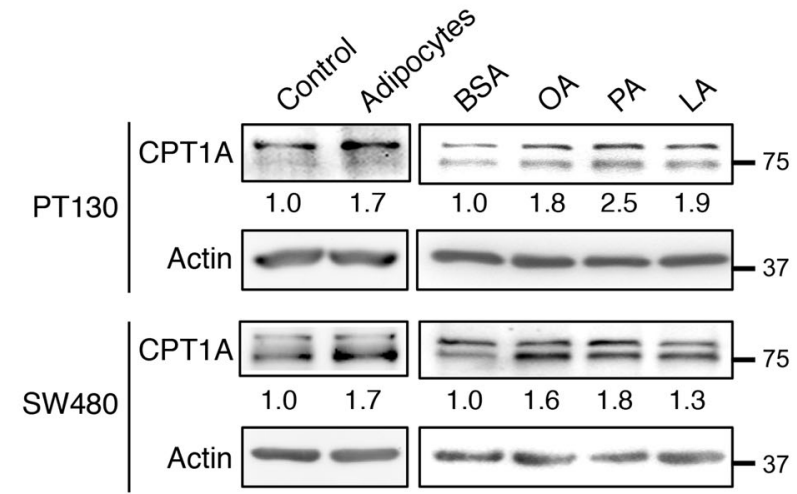

G

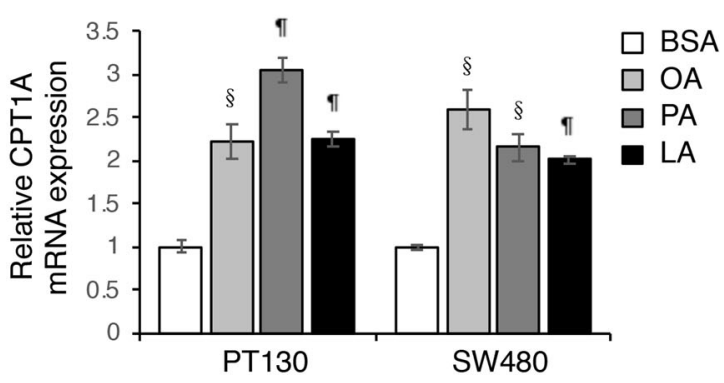

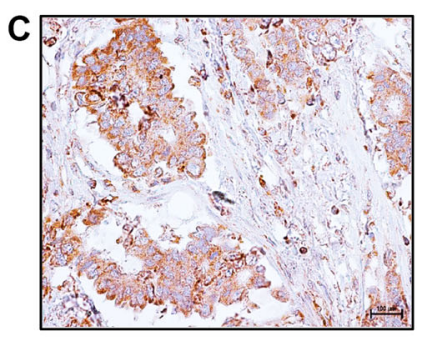

E

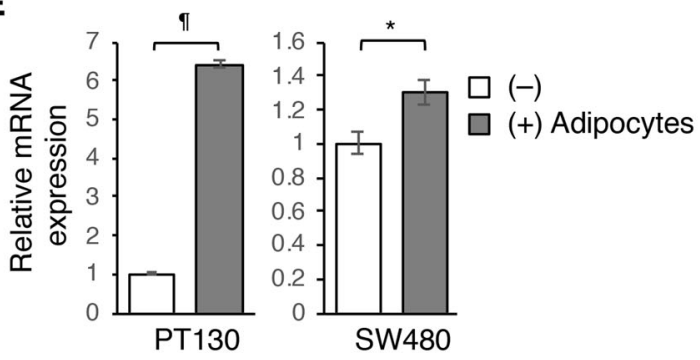

Fig. 1 The expression of CTP1A is upregulated by fatty acids in colon cancer. a-c The expression of CPT1A protein was detected in a stage IV colon cancer patient tissues using $\mathrm{IHC}$ staining. The black-boxed and blue-boxed regions shown in $\mathbf{a}$ were enlarged and presented in $\mathbf{b}$ and $\mathbf{c}$, respectively. Scale bar, $100 \mu \mathrm{m}$. d Quantitative analysis of CPT1A expression in specimens from 11 stage IV colon cancer patients. The percentage of invasive tumor cells in areas adjacent to adipocytes with positive CPT1A expression was higher than that of non-invasive primary tumors. Data represents mean \pm SD $\left(n=11,{ }^{*} p<0.01\right)$. e PT130 and SW480 cells were co-cultured with adipocytes isolated from colon cancer patients for 2 days. The levels of CPT1A mRNA expression were analyzed using RT-PCR. Data represents mean \pm SD $\left(n=3,{ }^{9} p<0.0001\right.$ and $\left.{ }^{*} p<0.01\right)$. f Protein lysates prepared from cells co-cultured with adipocytes as described in e or treated with BSA, OA, PA, and LA (100 $\mu$ M of each fatty acid species) for $24 \mathrm{~h}$ were analyzed for CPT1A protein expression using Western blotting. The relative levels of CPT1A were quantified by normalizing to $\beta$-actin and compared to control or BSA-treated cells. $\mathbf{g}$ PT130 and SW480 cells treated with BSA, OA, PA, or LA as described in $\mathbf{f}$ were analyzed for the expression of CPT1A mRNA using RT-PCR. Data represents mean \pm SD $\left(n=3,{ }^{8} p<0.001\right.$ and $\left.{ }^{n} p<0.0001\right)$. 
A
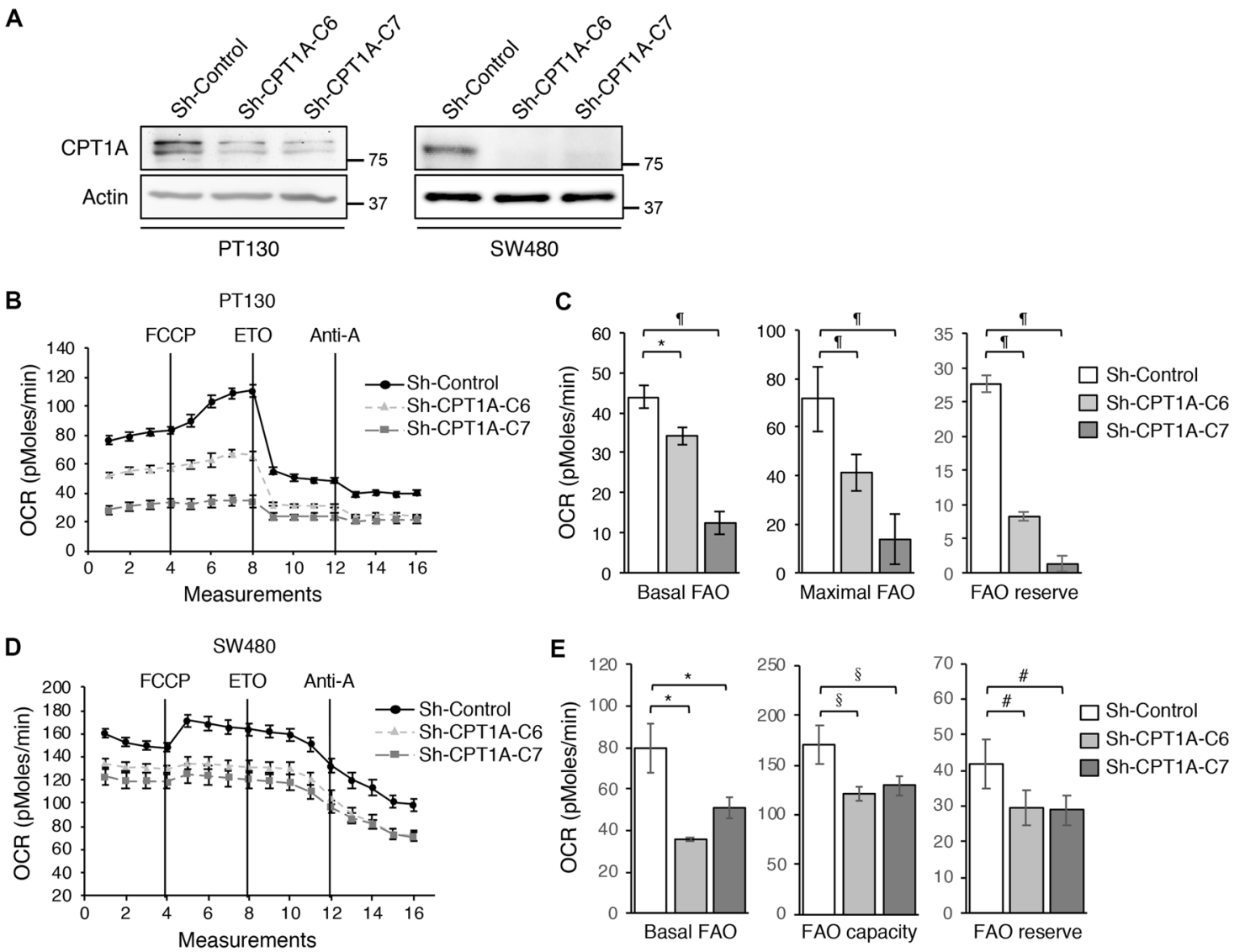

Fig. 2 Knockdown of CPT1A inhibits fatty acid oxidation in colon cancer cells. a The expression of CPT1A protein was analyzed in stable control and CPT1A knockdown PT130 and SW480 cells using Western blotting. Two different shRNA targeting sequences (C6 and C7) were used to silence CPT1A in each cell line. $\beta$-actin was used as loading controls. b Representative OCR measurements obtained from the FAO tests performed in control (sh-Control) and CPT1A knockdown (sh-CPT1A-C6 and sh-CPT1A-C7) PT130 cells using the Seahorse XF96 Extracellular Flux analyzer. FCCP, ETO, and antimycin $\mathrm{A}$ (Anti-A) were added at the indicated points. $\mathbf{c}$ Experiments as shown in $\mathbf{b}$ were quantified and the relative levels of OCR associated with basal FAO, FAO capacity, and FAO reserve were calculated based on the measurements obtained from the addition of different compounds. Data represent the mean \pm SD $\left(n=3, " p<0.0001\right.$ and $\left.{ }^{*} p<0.01\right)$. $\mathbf{d}$ Representative OCR measurements obtained from the FAO tests in sh-control, shCPT1A-C6, and sh-CPT1A-C7 SW480 cells using the Seahorse XF96 Extracellular Flux analyzer. e The relative levels of OCR associated with basal FAO, FAO capacity, and FAO reserve were quantified. Data represent the mean \pm SD $\left(n=3,{ }^{\#} p<0.05,{ }^{*} p<0.01\right.$, and $\left.{ }^{\S} p<0.001\right)$.

BODIPY-493/505 staining (Fig. 3a). However, while BODIPY-staining of lipid droplets diminished in the unloading phase in control cells, significant levels of BODIPY-staining remained in CPT1A knockdown cells (Fig. 3a). Moreover, the relative lipid content was quantified by measuring BODIPY fluorescence intensity at different time points following OA treatment. The time course of fatty acids degradation was significantly delayed in CPT1A knockdown PT130 and SW480 cells as indicated by increased BODIPY retention compared to control cells (Fig. 3b).

Furthermore, we determined if silencing CPT1A disrupts the protective effect provided by fatty acid uptake. Control and CPT1A knockdown PT130 and SW480 cells were co-cultured with adipocytes or treated with OA for $24 \mathrm{~h}$ and subsequently cultured in EBSS buffer for additional $48 \mathrm{~h}$ (Fig. 3c-f). Our results showed that both co-culturing with adipocytes and OA treatment significantly increased the survival of control PT130 and SW480 cells under nutrient deprivation conditions. However, the pro-survival effect provided by adipocytes and fatty acids was largely abolished in CPT1A knockdown cells (Fig. 3c-f). Taken together, our results demonstrate that uptake of fatty acids renders colon cancer cells resistant to nutrient deprivation and this acquired survival advantage relies on CPT1Amediated FAO.

\section{Downregulation of Cpt1a reduce cancer stem cell properties in 3D tumor organoids}

We have previously shown that the presence of adipocytes increases the expression of genes associated with 


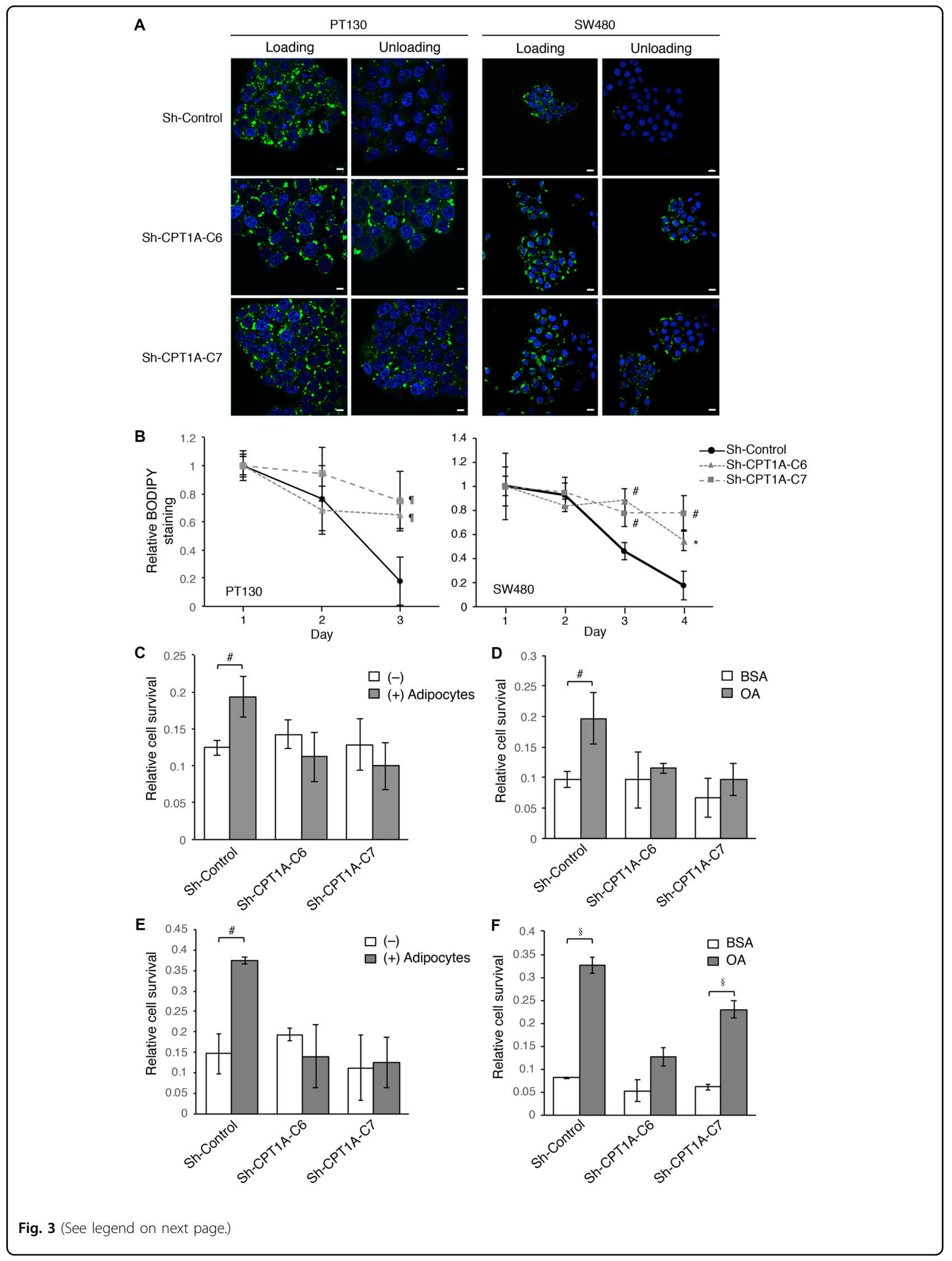


(see figure on previous page)

Fig. 3 Knockdown of CPT1A impairs fatty acid utilization and cell survival under nutrient deprivation conditions. a Stable control (shControl) and CPT1A knockdown (sh-CPT1A-C6 and sh-CPT1A-C7) PT130 and SW480 cells were incubated with OA for 24 h (loading) and subsequently allowed to grow in regular growth medium for additional 24 or $48 \mathrm{~h}$ for PT130 and SW480 cells, respectively (unloading). Representative confocal images of cells stained with BODIPY 493/503 (green) and DAPI (blue). Scale bar, 100 mm. b Control and CPT1A knockdown PT130 and SW480 cells were incubated with OA for $24 \mathrm{~h}$ (day 1) and subsequently were stained with BODIPY 493/503 at indicated time points (days 2-4). The fluorescence intensity was measured using a fluorescence spectrophotometer as readout for relative lipid contents in cells. Data represent the mean \pm SD $(n=3$, ${ }^{\#} p<0.05,{ }^{*} p<0.01$, and $" p<0.0001$ ). $\mathbf{c}$ and $\mathbf{d}$ Control and CPT1A knockdown PT130 cells were co-cultured with adipocytes $\mathbf{c}$ or pretreated with OA d for $24 \mathrm{~h}$ and subsequently cultured in EBSS for additional $48 \mathrm{~h}$. The relative cell survival was measured using crystal violet staining. Data represent the mean \pm SD $\left(n=3,{ }^{*} p<0.05\right)$. e and $\mathbf{f}$ Control and CPT1A knockdown SW480 cells were co-cultured with adipocytes e or pretreated with OA $\mathbf{f}$ for $24 \mathrm{~h}$ and subsequently cultured in EBSS for additional $48 \mathrm{~h}$. The relative cell survival was determined. Data represent the mean \pm SD $(n=3, \# p<0.05$ and $\left.{ }^{\S} p<0.001\right)$.

cancer stem cells in intestinal tumor organoids derived from Apc/Kras double mutant mice ${ }^{8}$. Here we investigated if upregulation of CPT1A is required to mediate the effect of adipocytes on promoting cancer stem cell functions. First, the Apc/Kras tumor organoids were embedded with adipocytes in 3D Matrigel or treated with different fatty acids and the expression of Cpt1A was monitored using Western blot and RT-PCR analysis. Consistent with results shown in Fig. 1, the presence of adipocytes or fatty acids increased Cpt1a expression at both protein and mRNA levels (Fig. 4a, b). Next, we silenced the expression of Cpt1a using lentiviral shRNA in Apc/Kras organoids. Interestingly, while control tumor cells formed spherical organoids in 3D, the Cptla knockdown organoids showed branched phenotype suggesting potential differentiation (Fig. 4c). Moreover, single cell suspensions of control and Cpt1a knockdown cells were seeded in 3D Matrigel and the numbers of colonies formed were determined after 6 days. We found that the ability of Cpt1a knockdown cells to form colonies in $3 \mathrm{D}$ was significantly decreased whereas the percentage of organoids with the branched phenotype was increased (Fig. 4d). Similar reduction in colony formation and increase in differentiation were observed in Apc/Kras organoids treated with ETO, an inhibitor of CPT1A (Supplementary Fig. S5A and B). In addition, since the 3D growth media are enriched in fatty acids, silencing Cpt1a also reduced the rate of cell proliferation in tumor organoids (Supplementary Fig. S5C). However, the reduced colony formation was not due to decreased cell survival as knockdown of Cpt1a had no effect on cell viability (Supplementary Fig. S5D).

Consistent with the notion that Wnt signaling activation is required to promote cancer stem cell properties ${ }^{23,24}$, we found that treating tumor organoids with three different fatty acids significantly increased expression of Wnt target genes and decreased genes associated with intestinal epithelial cell differentiation (Fig. 4e). Moreover, knockdown of Cpt1a largely reduced the expression of genes associated with CSCs downstream of Wnt/ $\beta$-catenin and increased the expression of differentiation markers (Fig. 4f). Furthermore, while fatty acid treatment significantly increased the expression of Wnt target genes, silencing Cpt1a expression abolished the effect of fatty acids (Fig. 4g). Collectively, our results suggest that fatty acid treatment enhances Wnt signaling in tumor organoids using a CPT1A-dependent mechanism.

\section{Fatty acids increase CPT1A expression and Wnt signaling through PPAR $\delta$ in colon cancer}

To determine the molecular mechanism by which fatty acids and adipocytes promote CPT1A expression, we investigated the functional contribution of peroxisome proliferator-activated receptor $\delta$ (PPAR $\delta$ ). PPAR $\delta$ is a lipid sensing nuclear receptor that can be activated by long-chain fatty acids to regulate cellular metabolism ${ }^{25}$. Previous studies have shown that PPAR $\delta$ play a critical role in controlling FAO in muscle or adipocytes tissues and high-fat diet enhance tumor initiation potential of intestinal organoids in by activating PPAR $\delta^{26,27}$. To confirm the involvement of PPAR $\delta$ in regulating CPT1A, we showed that treating PT130 cells or Apc/Kras tumor organoids with PPAR $\delta$ agonist GW501516 directly stimulated the expression of CPT1A and PPARGC1A (a known PPAR $\delta$ target gene) (Fig. 5a, b). In addition, fatty acid-induced upregulation of CPT1A was effectively blocked by pretreating cells with PPAR $\delta$ antagonist GSK3787 (Fig. 5c, d). Importantly, inhibition of fatty acidinduced PPAR $\delta$ activation decreased the expression of Wnt-targeting genes (Fig. 5e) in tumor organoids. Collectively, our data indicate that fatty acids stimulate CPT1A expression to enhance FAO and Wnt signaling mainly through PPAR $\delta$ activation in colon cancer cells.

\section{Downregulation of CPT1A decreases $\beta$-catenin acetylation and activation}

We next determined the mechanism underlying fatty acid-induced activation of Wnt signaling. The FAO has been identified as a major carbon source for the production of cellular Ac-CoA, a central metabolite that regulates protein acetylation ${ }^{16,28}$. Here we examined whether CPT1A downregulation alters cellular Ac-CoA levels. As shown in Fig. 6a, knockdown of CPT1A 

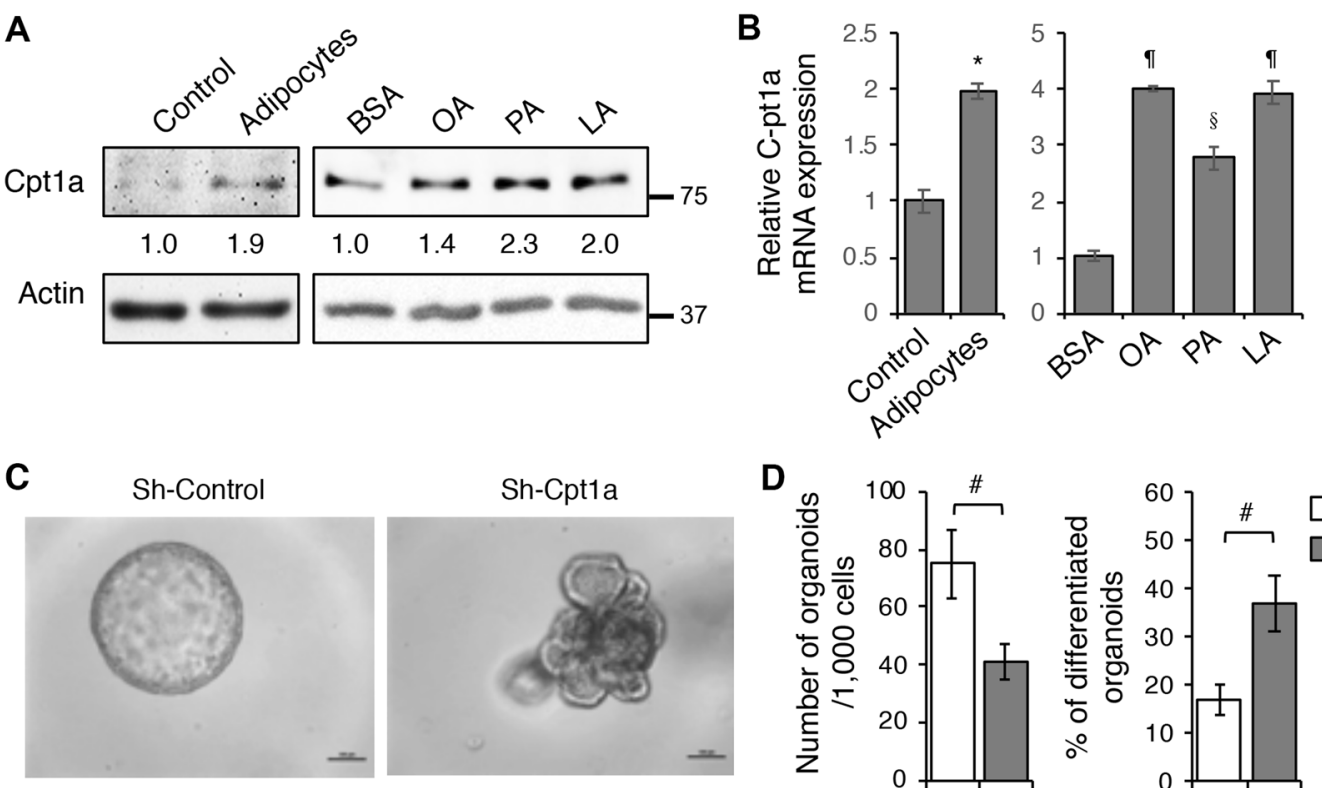

Sh-Cpt1a
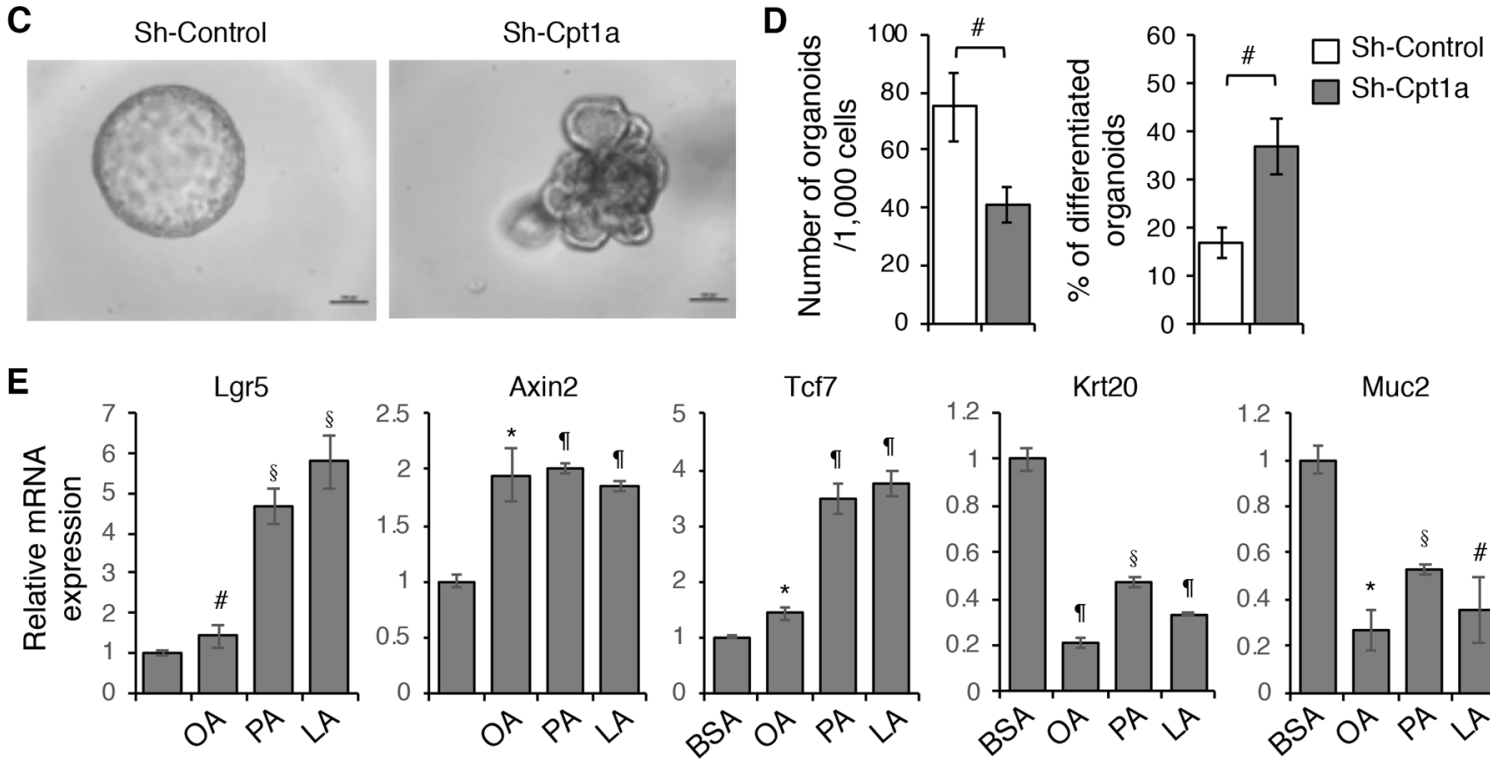

Axin2
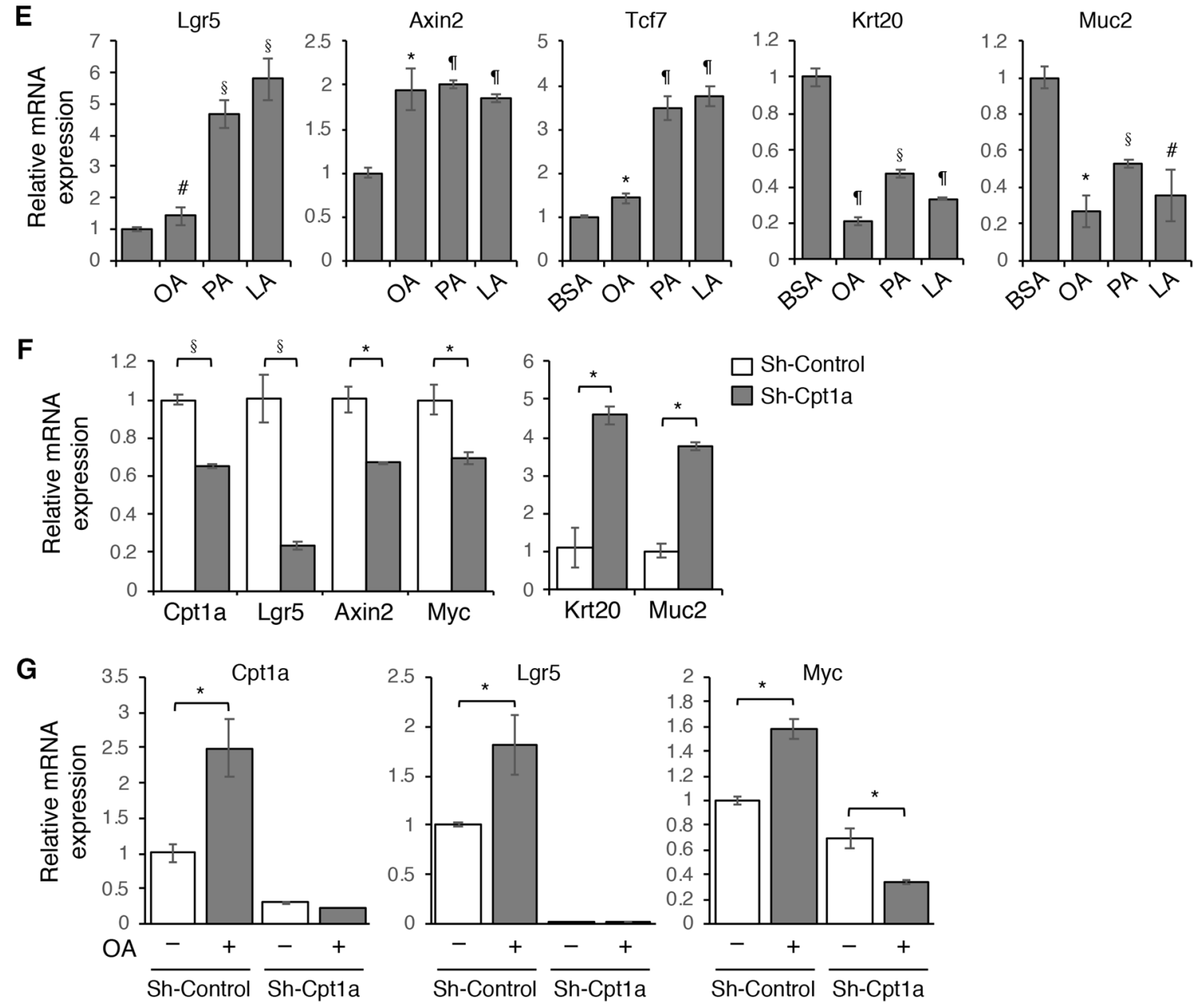

Fig. 4 (See legend on next page.) 
(see figure on previous page)

Fig. 4 CPT1A-dependent fatty acid oxidation promotes Wnt signaling and cancer stem cell properties. a Tumor organoids derived from Apc/ Kras double mutant mice were embedded with adipocytes in 3D Matrigel or treated with BSA, OA, PA, and LA (100 $\mu$ M each) for 2 days. The levels of Cpt1a expression were analyzed using Western blotting. The relative levels of CPT1A were quantified by normalizing to $\beta$-actin and compared to control or BSA-treated cells. $\mathbf{b}$ Tumor organoids treated as described in a were analyzed for the expression of Cpt1a mRNA using RT-PCR. Data represents mean \pm SD $\left(n=3,{ }^{*} p<0.01,{ }^{\$} p<0.001\right.$, and $\left.{ }^{\$} p<0.0001\right)$. $\mathbf{c}$ The expression of Cpt1a was silenced in Apc/Kras mutant tumor organoids using lentiviral shRNA. Single cell suspensions of control and Cpt1a knockdown cells were seeded in 3D Matrigel. Representative images of control and Cpt1a knockdown tumor organoids are shown after 6 days in culture. Scale bar, $100 \mu \mathrm{m}$. $\mathbf{d}$ The number of tumor organoids formed and the percentage of organoids showed branching phenotype were quantified (total 1000 cells were seeded per group). Data represent the mean \pm SD $\left(n=3,{ }^{\#} p<0.05\right)$. e Tumor organoids treated with BSA, OA, PA, and LA (100 $\mu \mathrm{M}$ each) were analyzed for the expression of Wnt $\beta$-catenin target genes (including Lgr5, Axin2, and Tcf7) and genes associated with intestinal epithelial cell differentiation (including Krt20 and Muc2) using RT-PCR. Data represents mean \pm SD $\left(n=3,{ }^{\#} p<0.05,{ }^{*} p<0.01,{ }^{\S} p<0.001\right.$, and $\left.{ }^{\$} p<0.0001\right)$. f Control and Cpt1a knockdown organoids were subseeded and grown in 3D Matrigel for 3 days. The expression of Cpt1a, Wnt/ $\beta$-catenin target genes (including Lgr5, Axin2, and Myc), and genes associated with intestinal epithelial cell differentiation (including Krt20 and Muc2) was determined using RT-PCR. Data represent the mean \pm SD $\left(n=3,{ }^{*} p<0.01\right.$ and

$\left.{ }^{\S} p<0.001\right)$. g Control and Cpt1a knockdown organoids were treated with OA for 2 days. The mRNA expression of Cpt1a as well as target genes of Wnt/ $\beta$-catenin (including Lgr5 and Myc) was determined using RT-PCR. Data represent the mean \pm SD $\left(n=3,{ }^{*} p<0.01\right)$.

significantly reduced total Ac-CoA levels in both PT130 and SW480 cells. Given that fatty acid-derived Ac-CoA can be used as substrate for protein acetylation and acetylation of $\beta$-catenin enhances Wnt signaling by promoting $\beta$-catenin nuclear localization ${ }^{29-31}$, we investigated whether the reduced acetyl-CoA production affects $\beta$-catenin activity. To this end, endogenous $\beta$-catenin proteins were immunoprecipitated from PT130 cells treated with BSA or OA and the level of $\beta$-catenin acetylation was determined using an acetylated-lysine antibody. Indeed, treatment with OA increased $\beta$-catenin acetylation (Fig. 6b). Moreover, knockdown of CPT1A markedly reduced levels of $\beta$-catenin acetylation in PT130 and SW480 cells suggesting CPT1A-dependent FAO controls $\beta$-catenin acetylation (Fig. 6c). Consistent with the notion that acetylation of $\beta$-catenin increases its activity, results from Western blot analysis showed that the levels of active $\beta$-catenin (non-phosphorylated form) were decreased in CPT1A knockdown PT130 and SW480 cells (Fig. 6d). As controls, we analyzed the acetylation status of $\alpha$-tubulin, a cytoplasmic substrate, and histone H3, a nuclear substrate, at lysine 9 (H3K9) and lysine 27 (H3K27) residues. Consistently, the levels of Ac- $\alpha-$ tubulin as well as H3K9Ac and H3K27Ac were reduced in CPT1A knockdown cells confirming that FAO is important for regulating a broad range of protein acetylation events (Fig. 6d). Similar decrease in the levels of active $\beta$-catenin and acetylated $\alpha$-tubulin, H3K9 and H3K27 was observed in Cpt1a knockdown tumor organoids (Supplementary Fig. S6A). To further determine the functional effect of decreased $\beta$-catenin acetylation, control and CPT1A knockdown PT130 and SW480 cells were fractionated into cytoplasmic and nuclear fractions. The amount of $\beta$-catenin in the nuclear factions was decreased in CPT1A knockdown PT130 and SW480 cells confirming that acetylation of $\beta$-catenin promotes its nuclear localization (Fig. 6e, f).
Furthermore, control and CPT1A knockdown PT130 cells treated with PA were subjected to Western blot analysis to determine the functional contribution of CPT1A-dependent FAO in regulating protein acetylation. Results showed that PA treatment increased levels of active $\beta$-catenin as well as acetylated $\alpha$-tubulin, H3K9, and H3K27 in control cells whereas silencing CPT1A blocked the effect of fatty acids on $\beta$-catenin activation and protein acetylation (Fig. 6g). Similar results were obtained in control and CPT1A knockdown cells treated with OA (Supplementary Fig. S6B). As a control, we treated cells with octanoate, a medium-chain fatty acid that can enter mitochondria and undergo FAO independent of $\mathrm{CPT}_{1 \mathrm{~A}}{ }^{16}$. Interestingly, octanoate treatment markedly increased the levels of acetylated $\alpha$-tubulin, H3K9, and H3K27 as well as active $\beta$-catenin in both control and CPT1A knockdown cells (Fig. 6h). Consistent with a dosedependent effect of octanoate on modulating protein acetylation ${ }^{16}$, we showed that treating cells with a lower concentration of octanoate partially rescued the acetylation defect observed in CPT1A knockdown cells (Supplementary Fig. S6C). Taken together, our results suggest the acetyl-CoA derived from long-chain FAO can be used as the substrate for protein acetylation in a CPT1Adependent manner. Because free fatty acids derived from adipocytes are in the form of long-chain fatty acids, upregulation of CPT1A allows the cancer cells to produce increasing levels of Ac-CoA via mitochondrial FAO to promote $\beta$-catenin activation and Wnt signaling.

\section{Knockdown of CPT1A inhibits xenograft tumor growth and tumor initiation in vivo}

To examine the effect of silencing CPT1A on tumor growth in vivo, we subcutaneously injected control and CPT1A knockdown SW480 cells mixed with Matrigel alone or in combination with human adipocytes into NSG mice and monitor the tumorigenesis process. Consistently with the tumor promoting effect of adipocytes, the rate of 


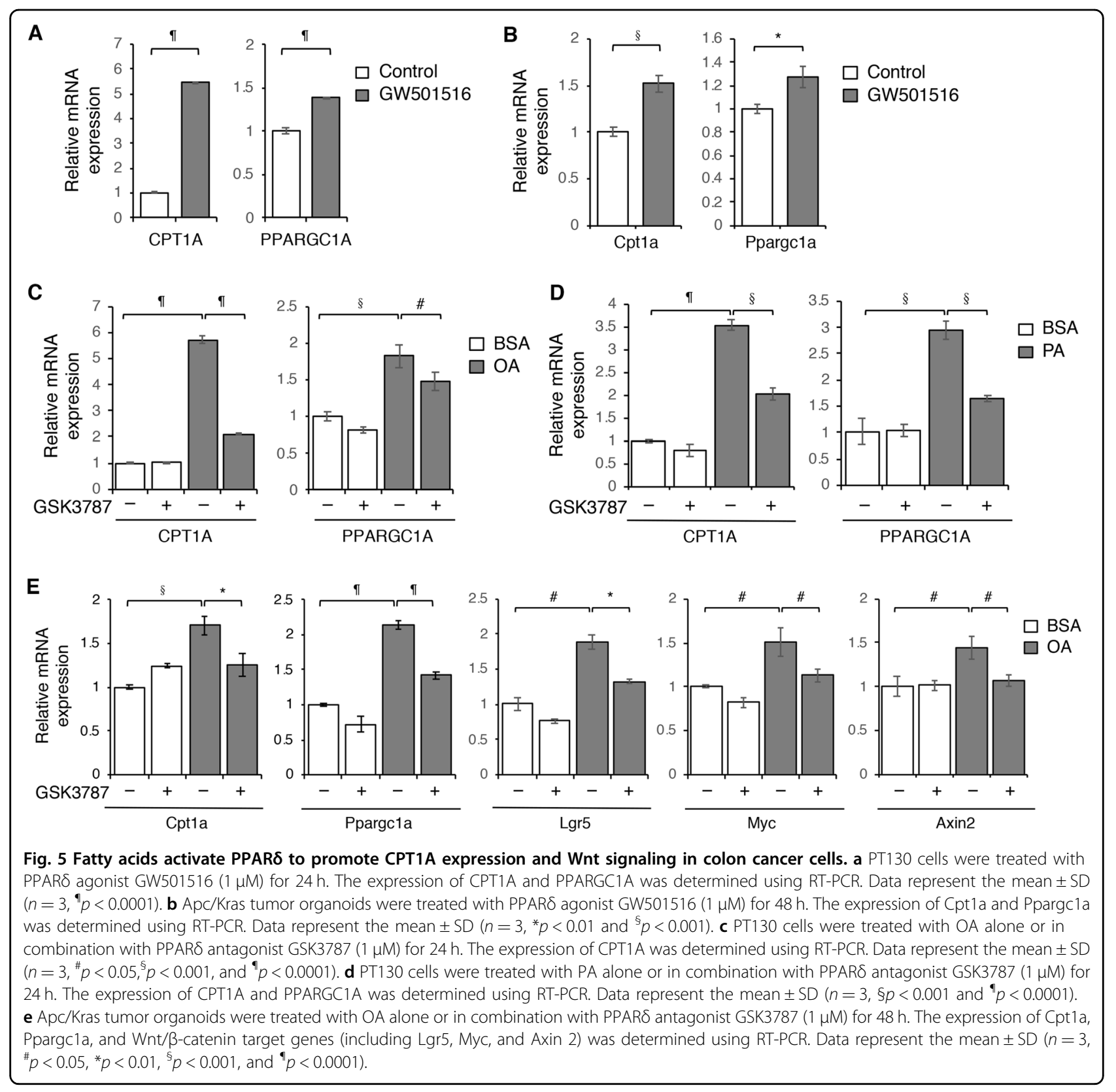

tumor growth was largely increased when the control SW480 cells were co-injected with adipocytes (Fig. 7a). Although knockdown of CPT1A did not significantly decrease the tumor growth rate basally compared to the control group, CPT1A-loss blocked the tumor promoting effect of adipocytes in vivo (Fig. 7a). Analysis of tumor tissues collected from four groups of mice revealed that knockdown of CPT1A decreased the amount of active $\beta$-catenin, although we did not observe the adipocyteinduced activation of $\beta$-catenin in tumors derived from control cells (Fig. 7b, c). It is likely that the effect of adipocytes on promoting protein acetylation gradually diminished as adipocytes did not expect to survive the entire tumorigenesis process. Nevertheless, results from RT-PCR analysis showed that the expression of CPT1A and Wnt target genes (including LGR5 and MYC) was elevated in control SW480 cells that co-injected with adipocytes and knockdown of CPT1A largely abolished this effect (Fig. 7d).

Furthermore, we performed tumor initiation experiments by injecting control and CPT1A knockdown SW480 cells mixed with Matrigel alone or in combination with adipocytes into NSG mice at 100 and 1000 cells per site. The number of tumors formed was determined after 3 months. Based on an ELDA, co-injection of adipocytes significantly increased the stem cell frequency in control 


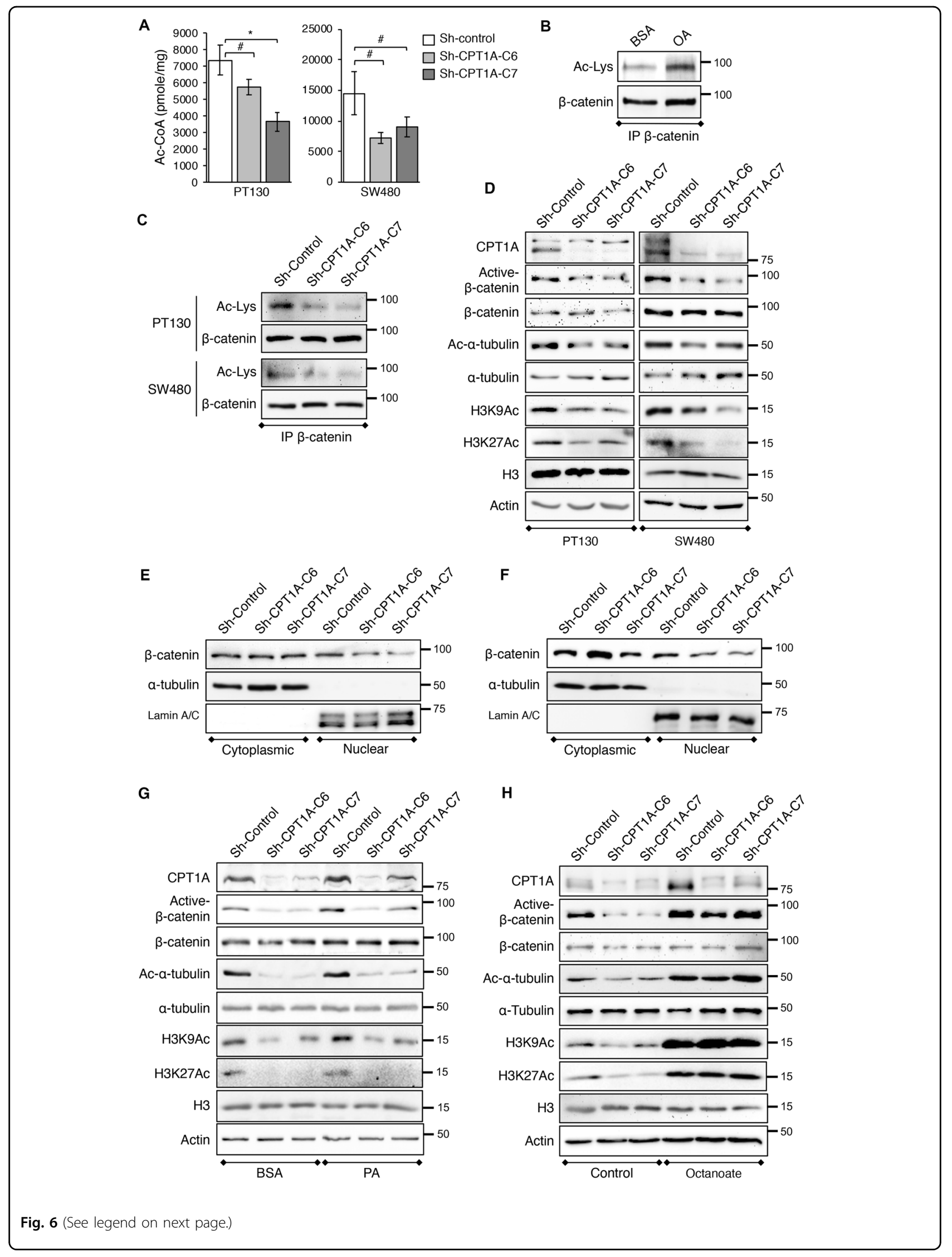


(see figure on previous page)

Fig. 6 Downregulation of CPT1A decreases cellular levels of acetyl-CoA and protein acetylation. a Control and CPT1A knockdown PT130 and SW480 cells were cultured in low glucose media supplemented with 10\% FBS. The levels of acetyl-CoA in control and CPT1A knockdown PT130 and SW480 cells were determined using the Acetyl-CoA Assay kit. Data represent mean \pm SD $\left(n=3,{ }^{*} p<0.05\right.$ and $\left.{ }^{*} p<0.01\right)$. b PT130 cells were treated with BSA or OA $(100 \mu \mathrm{M})$ for $24 \mathrm{~h}$. Cell lysates were immunoprecipitated using the anti- $\beta$-catenin antibody and analyzed for the levels of $\beta$-catenin acetylation using the acetylated-lysine antibody (Ac-Lys). The blot was stripped and reprobed for total $\beta$-catenin in the immunoprecipitates. c Cell lysates from control and CPT1A knockdown PT130 or SW480 cells were immunoprecipitated and analyzed for the levels of $\beta$-catenin acetylation using the acetylated-lysine antibody (Ac-Lys). The blot was stripped and reprobed for total $\beta$-catenin in the immunoprecipitates. $\mathbf{d}$ Cell lysates from control and CPT1A knockdown PT130 or SW480 cells were analyzed for the levels of active- $\beta$-catenin as well as acetyl-a-tubulin (Ac-a-tubulin), acetylhistone at K9 and K27 residues (H3K9Ac and H3K27Ac) using Western blotting. Total $\beta$-catenin, $a$-tubulin, histone H3, and $\beta$-actin were used as loading controls. e and $\mathbf{f}$ Control and CPT1A knockdown PT130 e and SW480 f cells were fractionated into cytoplasmic and nuclear fractions. The amount of $\beta$-catenin in each fraction was determined using the anti- $\beta$-catenin antibody. a-Tubulin and lamin A/C were used as controls for the cytoplasmic and nuclear fractions, respectively. $\mathbf{g}$ Control and CPT1A knockdown PT130 cells were treated with BSA or PA (100 $\mu$ M) for $24 \mathrm{~h}$ and cell lysates were analyzed for the levels of active- $\beta$-catenin as well as Ac-a-tubulin, H3K9Ac, and H3K27Ac using Western blotting. $\mathbf{h}$ Control and CPT1A knockdown PT130 cells were cultured under control condition or treated with octanoate $(3 \mathrm{mM})$ for $24 \mathrm{~h}$ and cell lysates were analyzed for the levels of active- $\beta$-catenin as well as Ac-a-tubulin, H3K9Ac, and H3K27Ac using Western blotting.

cells (from 1 in 458 basally to 1 in 102 in the presence of adipocytes) but not in CPT1A knockdown cells (Fig. 7e). Taken together, our results suggest that CPT1A is required for adipocytes to enhance the tumor initiation potential in vivo.

In summary, results from this study support a model in which uptake of fatty acids activates PPAR $\delta$-dependent transcription of CPT1A and CPT1A-dependent FAO. Subsequently, increased production of Ac-CoA and the acetylation of $\beta$-catenin promote $\mathrm{Wnt}$ signaling and cancer stem cell function. Thus, pharmacological inhibition of CPT1A with ETO or knockdown of CPT1A expression may block fatty acids-induced tumor promoting effects in colon cancer (Supplementary Fig. S6D).

\section{Discussion}

Several large prospective epidemiological studies have provided strong evidence supporting the role of obesity in promoting colon cancer initiation and progression ${ }^{5,32,33}$. However, the molecular mechanism underlying how adipose tissue and adipocytes support tumor growth and progression remains largely unknown. We have shown previously that abundant adipocytes are found in direct contact with colon cancer cells and uptake of fatty acids released by adipocytes promotes cancer cell survive by upregulating mitochondrial $\mathrm{FAO}^{8}$. Results from this study demonstrate that the presence of adipocytes or fatty acids stimulates the expression of CPT1A by activating PPAR $\delta$ dependent transcription. Silencing CPT1A expression in colon cancer cells blocks the cell survival advantage provided by adipocytes as a result of decreased fatty acid degradation via FAO. In addition, CPT1A downregulation induces differentiation of tumor organoids grown in 3D and attenuates the effect of fatty acids on promoting the expression of cancer stem cell-associated genes. Importantly, CPT1A expression is required for adipocytes to promote tumor growth and initiation in vivo.
The cancer stem cells, also known as tumor-initiating cells, have been implicated in tumor initiation, recurrence, and metastasis ${ }^{34-36}$. Emerging evidence suggests that $\mathrm{Wnt} / \beta$-catenin signaling is required for the maintenance of normal and cancer stem cells ${ }^{37}$. Despite having activating mutations in the Wnt pathway, colon cancer cells with the highest levels of $\beta$-catenin signaling display cancer stem cell properties ${ }^{23}$. Consequently, the expression levels of $\beta$-catenin target genes have been used as a readout for the stemness of cancer stem cells ${ }^{38}$. Recent studies have begun to establish the role of FAO in regulating normal and cancer stem cell function. For example, it has been shown that CPT1A-dependent FAO is required for the maintenance of tumor initiation cells in hepatocellular carcinoma as well as normal hematopoietic and neural stem cells ${ }^{39-41}$. Moreover, deletion of Cpt1a in mouse intestine epithelium decreases the number and function of normal intestinal stem cells ${ }^{42}$. Our study identifies CPT1A upregulation as a key metabolic alteration that cancer cells adapt to promote $\beta$-catenin acetylation and activation in an adipocyte-enriched TME. Since uptake of fatty acids induces the acetylation of proteins other than $\beta$-catenin, additional studies are needed to determine the functional contribution of these acetylation events. It is of particular interest to further investigate the epigenetic alterations associated with histone acetylation changes induced by fatty acids.

In addition, it has been shown recently that CPT1 complex may support cell proliferation independent of its ability to control FAO ${ }^{43}$. Although we show that inhibition of FAO is coupled with decreased cell survival and $\beta$-catenin activation in CPT1A knockdown cells, it is possible that CPT1A mediates the tumor promoting effects of adipocytes using a FAO-independent mechanism. Moreover, a recent study reported that circulating fatty acid-binding protein released by adipose tissue (A-FABP or FABP4) promotes breast cancer stemness by activating the IL-6/STAT3/ALDH1 pathway ${ }^{44}$. The 

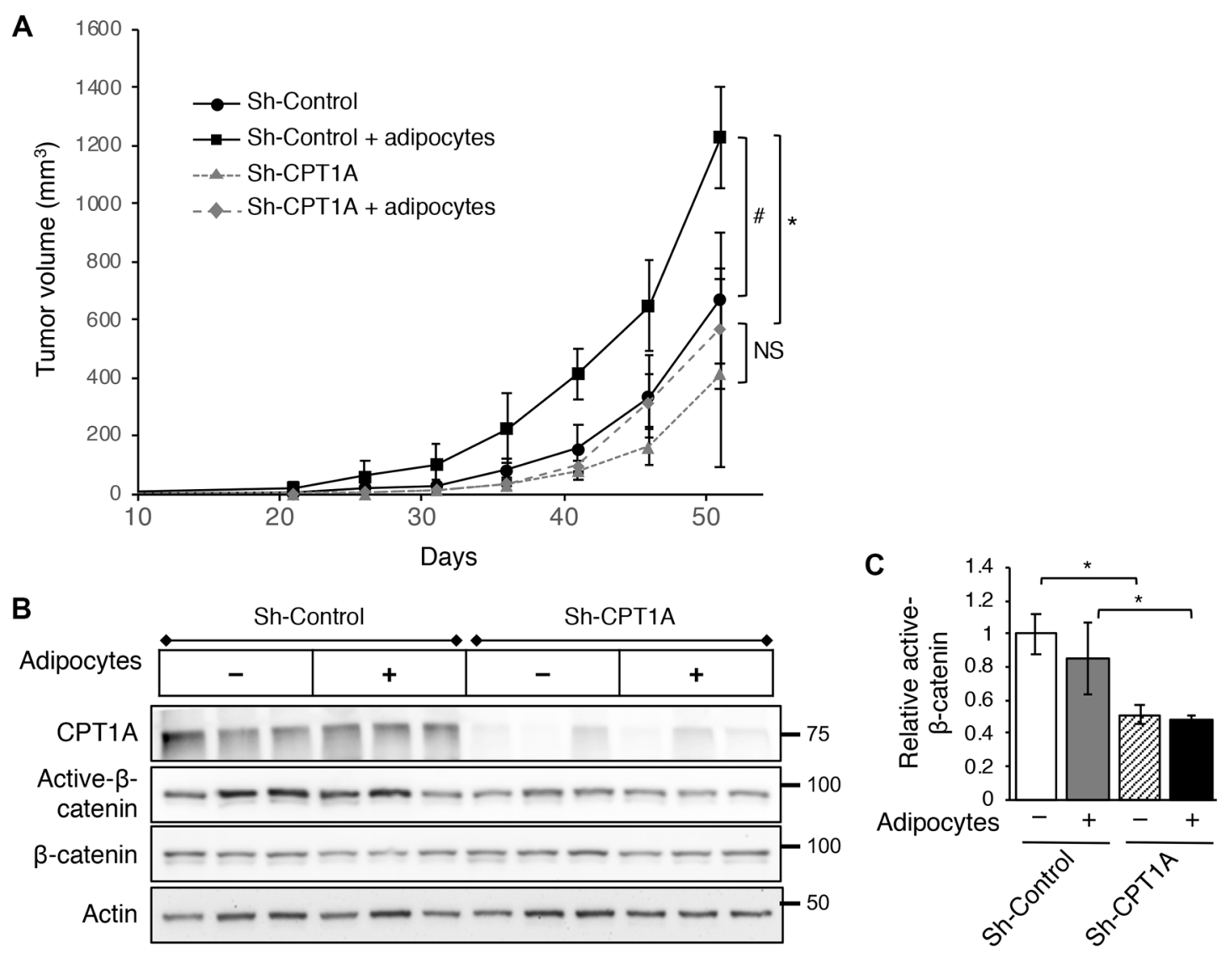

D

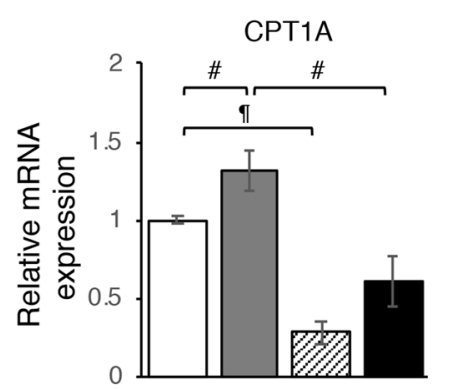

Adipocytes
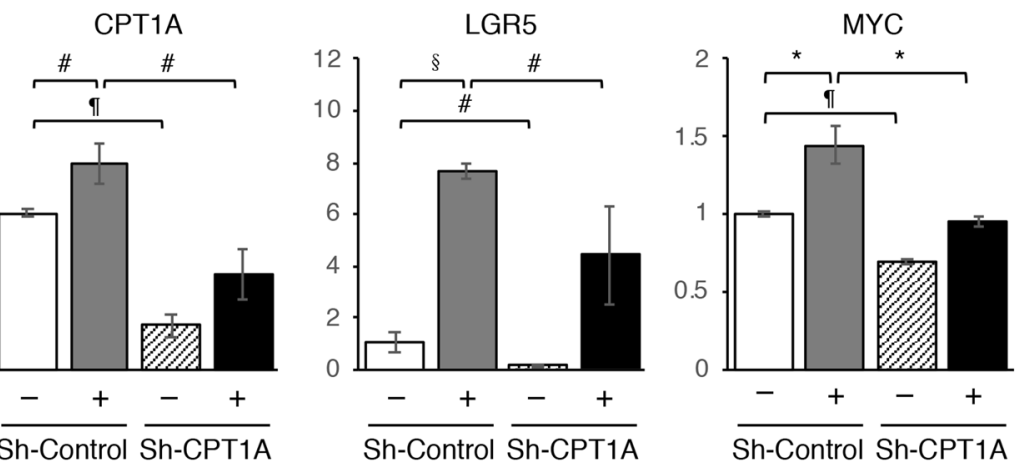

E

\begin{tabular}{|c|c|c|c|c|c|}
\hline \multicolumn{2}{|c|}{} & \multicolumn{2}{c|}{ Sh-Control } & \multicolumn{2}{c|}{ Sh-CPT1A } \\
\hline \multicolumn{2}{|c|}{ Adipocytes } & - & + & - & + \\
\hline \multirow{2}{*}{$\begin{array}{c}\text { Number of } \\
\text { cells/injection }\end{array}$} & 1,000 & $6 / 8$ & $8 / 8$ & $5 / 8$ & $5 / 8$ \\
\cline { 2 - 6 } & 100 & $4 / 8$ & $5 / 8$ & $2 / 8$ & $1 / 8$ \\
\hline \multicolumn{2}{|c|}{ Estimated stem cell frequency } & $458^{\&}$ & 102 & 814 & $971^{* *}$ \\
\hline
\end{tabular}

Fig. 7 (See legend on next page.) 
(see figure on previous page)

Fig. 7 CPT1A is required to mediate the tumor promoting effect of adipocytes in vivo. a Control and CPT1A knockdown SW480 cells were mixed with Matrigel alone or in combination with adipocytes and injected subcutaneously into NSG mice. The size of the tumors was measured every 5 days for 51 days. Data represent the mean \pm SEM $(n=6$, for sh-Control and sh-CPT1A group; and $n=7$ for sh-Control + adipocytes and shCPT1A + adipocytes group, ${ }^{\#} p<0.05$ and ${ }^{*} p<0.01 ; N S=$ not significant). $\mathbf{b}$ Tumor tissues from three mice of each group were analyzed for the levels of CPT1A, active- $\beta$-catenin, and $\beta$-catenin using Western blotting. c Quantitative analysis of relative active- $\beta$-catenin levels in xenograft tumors from four different groups of mice. The levels of active- $\beta$-catenin were normalized to total $\beta$-catenin in each sample. Data represent the mean \pm SD $(n=3$, $\left.{ }^{*} p<0.01\right)$. d Tumor tissues from three mice of each group were analyzed for the expression of CPT1A, LGR5, and MYC using RT-PCR. Data represent the mean $\pm \mathrm{SD}\left(n=3,{ }^{*} p<0.05,{ }^{*} p<0.01, \S p<0.001\right.$, and $\left.{ }^{\natural} p<0.0001\right)$. e Tumor initiation experiments were performed using control and CPT1A knockdown SW480 cells. Cells were mixed with Matrigel alone or in combination with adipocytes and injected into NSG mice at 100 or 1000 cells per site and total eight injections were used for each cell group. The number of tumors formed was determined 3 months post inoculation. The stem cell frequency was calculated using extreme limiting dilution analysis (ELDA) $\& p=0.01$, comparing sh-control group with sh-control + adipocytes group; and ${ }^{* *} p=0.0003$, comparing sh-control + adipocytes group with sh-CPT1A + adipocytes group).

importance of FABP4 in facilitating the transport of fatty acids from adipocytes to cancer cells has also been shown in ovarian and colon cancers ${ }^{7,8}$. Results from our study here provide strong evidence supporting a role of CPT1A in controlling the production of signaling metabolites to mediate the communication between adipocytes and colon cancer cells. Future studies are needed to further determine the role of FABP4 in regulating cancer stemness in colon cancer.

In summary, results from our study identify CPT1A as a key regulator that connects adipocyte-mediated regulation of cellular metabolism to Wnt signaling in colon cancer cells. Given the preferential upregulation of CPT1A by adipocytes, our findings indicate that inhibition of CPT1A may provide an effective approach to block the tumor promoting effects of adipocytes in colon cancer.

\section{Acknowledgements}

This work was supported by R01CA133429 (T.G.), R01CA208343 (B.M.E. and T.G.) and a pilot grant from P20GM121327 (University of Kentucky Center for Cancer and Metabolism). The studies were conducted with support provided by the Redox Metabolism, Biospecimen Procurement and Translational Pathology, Cancer Research Informatics, Flow Cytometry and Immune Monitoring, and Biostatistics and Bioinformatics Shared Resource Facilities of the University of Kentucky Markey Cancer Center (P30CA177558) at the University of Kentucky.

\section{Author details}

'Markey Cancer Center, University of Kentucky, Lexington, KY 40536-0679, USA. ${ }^{2}$ Department of Toxicology and Cancer Biology, University of Kentucky, Lexington, KY 40536-0679, USA. ${ }^{3}$ Department of Surgery, University of Kentucky, Lexington, KY 40536-0679, USA. ${ }^{4}$ Department of Molecular and Cellular Biochemistry, University of Kentucky, Lexington, KY 40536-0679, USA

\section{Conflict of interest}

The authors declare that they have no conflict of interest.

\section{Publisher's note}

Springer Nature remains neutral with regard to jurisdictional claims in published maps and institutional affiliations.

Supplementary Information accompanies this paper at (https://doi.org/ 10.1038/s41419-020-02936-6).

Received: 17 January 2020 Revised: 21 August 2020 Accepted: 27 August 2020

Published online: 10 September 2020

\section{References}

1. Cairns, R. A., Harris, I., McCracken, S. \& Mak, T. W. Cancer cell metabolism. Cold Spring Harb. Symp. Quant. Biol. 76, 299-311 (2011).

2. Ward, P. S. \& Thompson, C. B. Metabolic reprogramming: a cancer hallmark even Warburg did not anticipate. Cancer Cell 21, 297-308 (2012).

3. Anastasiou, D. Tumour microenvironment factors shaping the cancer metabolism landscape. Br. J. Cancer 116, 277-286 (2017).

4. Pearce, O. M. T. et al. Deconstruction of a metastatic tumor microenvironment reveals a common matrix response in human cancers. Cancer Discov. 8, 304-319 (2018).

5. Nieman, K. M., Romero, I. L., Van Houten, B. \& Lengyel, E. Adipose tissue and adipocytes support tumorigenesis and metastasis. Biochim. Biophys. Acta 1831, 1533-1541 (2013).

6. Park, J., Morley, T. S., Kim, M., Clegg, D. J. \& Scherer, P. E. Obesity and cancermechanisms underlying tumour progression and recurrence. Nat Rev Endocrinol 10, 455-465 (2014).

7. Nieman, K. M. et al. Adipocytes promote ovarian cancer metastasis and provide energy for rapid tumor growth. Nat. Med. 17, 1498-1503 (2011).

8. Wen, Y. A. et al. Adipocytes activate mitochondrial fatty acid oxidation and autophagy to promote tumor growth in colon cancer. Cell Death Dis. 8, e2593 (2017).

9. Wang, Y. Y. et al. Mammary adipocytes stimulate breast cancer invasion through metabolic remodeling of tumor cells. JCI Insight 2, e87489 (2017).

10. Zhang, M. et al. Adipocyte-derived lipids mediate melanoma progression via FATP proteins. Cancer Discov. 8, 1006-1025 (2018).

11. Batlle, E. \& Clevers, H. Cancer stem cells revisited. Nat. Med. 23, 1124-1134 (2017).

12. Shimokawa, M. et al. Visualization and targeting of LGR5(+) human colon cancer stem cells. Nature 545, 187-192 (2017).

13. de Sousa e Melo, F. et al. A distinct role for $\operatorname{Lgr} 5(+)$ stem cells in primary and metastatic colon cancer. Nature 543, 676-680 (2017).

14. Wen, Y. A. et al. Downregulation of SREBP inhibits tumor growth and initiation by altering cellular metabolism in colon cancer. Cell Death Dis. 9, 265 (2018).

15. Abdelmagid, S. A. et al. Comprehensive profiling of plasma fatty acid concentrations in young healthy Canadian adults. PLOS ONE 10, e0116195 (2015).

16. McDonnell, E. et al. Lipids reprogram metabolism to become a major carbon source for histone acetylation. Cell Rep. 17, 1463-1472 (2016).

17. Liu, J. et al. Loss of PHLPP expression in colon cancer: role in proliferation and tumorigenesis. Oncogene 28, 994-1004 (2009).

18. Xiong, X. et al. PHLPP regulates hexokinase 2-dependent glucose metabolism in colon cancer cells. Cell Death Discov. 3, 16103 (2017).

19. Hu, Y. \& Smyth, G. K. ELDA: extreme limiting dilution analysis for comparing depleted and enriched populations in stem cell and other assays. J. Immunol. Methods 347, 70-78 (2009).

20. Iggman, D., Arnlov, J., Cederholm, T. \& Riserus, U. Association of adipose tissue fatty acids with cardiovascular and all-cause mortality in elderly men. JAMA Cardiol. 1, 745-753 (2016).

21. Lionetti, V., Stanley, W. C. \& Recchia, F. A. Modulating fatty acid oxidation in heart failure. Cardiovasc. Res. 90, 202-209 (2011).

22. Schlaepfer, I. R. et al. Lipid catabolism via CPT1 as a therapeutic target for prostate cancer. Mol. Cancer Ther. 13, 2361-2371 (2014). 
23. Vermeulen, L. et al. Defining stem cell dynamics in models of intestinal tumor initiation. Science 342, 995-998 (2013).

24. Vermeulen, L. et al. Wnt activity defines colon cancer stem cells and is regulated by the microenvironment. Nat. Cell Biol. 12, 468-476 (2010).

25. Reilly, S. M. \& Lee, C. H. PPAR delta as a therapeutic target in metabolic disease. FEBS Lett. 582, 26-31 (2008).

26. Furnsinn, C., Willson, T. M. \& Brunmair, B. Peroxisome proliferator-activated receptor-delta, a regulator of oxidative capacity, fuel switching and cholesterol transport. Diabetologia 50, 8-17 (2007).

27. Beyaz, S. et al. High-fat diet enhances stemness and tumorigenicity of intestinal progenitors. Nature 531, 53-58 (2016).

28. Pietrocola, F., Galluzzi, L., Bravo-San Pedro, J. M., Madeo, F. \& Kroemer, G. Acetyl coenzyme A: a central metabolite and second messenger. Cell Metab. 21, 805-821 (2015).

29. Chocarro-Calvo, A., Garcia-Martinez, J. M., Ardila-Gonzalez, S., De la Vieja, A. \& Garcia-Jimenez, C. Glucose-induced beta-catenin acetylation enhances Wnt signaling in cancer. Mol. Cell 49, 474-486 (2013).

30. Levy, L. et al. Acetylation of beta-catenin by p300 regulates beta-catenin-Tcf4 interaction. Mol. Cell Biol. 24, 3404-3414 (2004).

31. Hoffmeyer, K., Junghans, D., Kanzler, B. \& Kemler, R. Trimethylation and acetylation of beta-catenin at lysine 49 represent key elements in ESC pluripotency. Cell Rep. 18, 2815-2824 (2017).

32. Yehuda-Shnaidman, E. \& Schwartz, B. Mechanisms linking obesity, inflammation and altered metabolism to colon carcinogenesis. Obes. Rev. 13 1083-1095 (2012).

33. Calle, E. E., Rodriguez, C., Walker-Thurmond, K. \& Thun, M. J. Overweight, obesity, and mortality from cancer in a prospectively studied cohort of U.S. adults. N. Engl. J. Med. 348, 1625-1638 (2003).
34. Kreso, A. et al. Self-renewal as a therapeutic target in human colorectal cancer Nat. Med. 20, 29-36 (2014).

35. Kreso, A. et al. Variable clonal repopulation dynamics influence chemotherapy response in colorectal cancer. Science 339, 543-548 (2013).

36. Merlos-Suarez, A. et al. The intestinal stem cell signature identifies colorectal cancer stem cells and predicts disease relapse. Cell Stem Cell 8 , 511-524 (2011).

37. Clevers, H. Wnt/beta-catenin signaling in development and disease. Cell 127, 469-480 (2006).

38. Zeuner, A., Todaro, M., Stassi, G. \& De Maria, R. Colorectal cancer stem cells: from the crypt to the clinic. Cell Stem Cell 15, 692-705 (2014).

39. Chen, C. L. et al. NANOG metabolically reprograms tumor-initiating stem-like cells through tumorigenic changes in oxidative phosphorylation and fatty acid metabolism. Cell Metab. 23, 206-219 (2016).

40. Ito, K. et al. A PML-PPAR-delta pathway for fatty acid oxidation regulates hematopoietic stem cell maintenance. Nat. Med. 18, 1350-1358 (2012).

41. Knobloch, M. et al. A fatty acid oxidation-dependent metabolic shift regulates adult neural stem cell activity. Cell Rep. 20, 2144-2155 (2017).

42. Mihaylova, M. M. et al. Fasting activates fatty acid oxidation to enhance intestinal stem cell function during homeostasis and aging. Cell Stem Cell 22 769-778 e764 (2018).

43. Yao, C. H. et al. Identifying off-target effects of etomoxir reveals that carnitine palmitoyltransferase I is essential for cancer cell proliferation independent of beta-oxidation. PLoS Biol. 16, e2003782 (2018).

44. Hao, J. et al. Circulating adipose fatty acid binding protein is a new link underlying obesity-associated breast/mammary tumor development. Cell Metab. 28, 689-705.e685 (2018). 